\title{
Einleitung: „Intelligenz ist nicht das Privileg von Auserwählten."
}

\author{
Marc Bovenschulte, Julian Stubbe
}

Im Jahr 1987 formulierte Victor Serebriakoff, britischer Autor und ehemaliger Präsident der Gesellschaft für Menschen mit hohem IQ "Mensa”, bei seiner Reflexion über die biologische und die - zu diesem Zeitpunkt noch recht überschaubare - Kl, dass Intelligenz stets ihre eigene Vervollkommnung anstrebe. Serebriakoff betrachtete den Menschen dabei nicht als eine Spezies von Auserwählten, der das Recht vorbehalten sei, intelligent zu sein gegenüber einer profanen, nicht-intelligenten Umwelt. Vielmehr verstand er Intelligenz als etwas Universelles, das sich über unterschiedliche Substrate hinweg infolge von neuen, sich stetig verändernden Informationen optimiert. So gesehen lässt sich die Evolution des Lebens auch als eine der Intelligenz verstehen. Sie strebt an, sich unabhängig vom Lebewesen oder vom materiellen Substrat weiterzuentwickeln. Nach Serebriakoff ist es nur logisch, dass die Intelligenz eine Erweiterung ihrer Fähigkeiten durch Erweiterung ihrer materiellen Basis sucht - also vom Neuron zum Chip. Die KI ist damit unter Umständen eine Erweiterung oder Fortsetzung der natürlichen Intelligenz, sie stellt keine Konkurrenz und damit auch nicht per se eine Bedrohung dar.

Serebriakoffs Perspektive lädt ein, das Verhältnis von KI und Gesellschaft neu zu überdenken. Sie lehnt es ab, Kategorien als Dichotomie zu behandeln, als zwei getrennte Sphären, die nur vereinzelt aufeinander treffen, also etwa „die Gesellschaft hier" und "die Technologie dort". Im Gegenteil: Nach seiner Auffassung sind Innovationen, Neuerungen oder Wandlungen als Kontinuum zu betrachten, sowohl zeitlich als auch im Hinblick auf aktuelle Zusammenhänge zwischen Gesellschaft, Politik, Wissenschaft und Technik. Alle diese Bereiche entwickeln sich sowohl auf Pfaden, die von einer gewissen Eigendynamik geprägt sind, als auch in stetiger Wechselbeziehung zueinander. Veränderungen und Disruptionen, also Zerrüttungen von gegebenen Strukturen, erscheinen aus dieser Sicht nicht als gleichsam kosmische Ereignisse, sondern sind vielmehr, wie in der biologischen Evolution, Ergebnisse kontinuierlicher Mutation und Selektion. Durch diese entsteht Neues, einzelne Innovationen setzen sich durch und werden als funktionierende Technik zur Grundlage für Folgeinnovationen.

Des Weiteren lehrt er uns etwas über die Beziehung des Menschen - in seiner Natur als reflexives Wesen - zur KI, und das insbesondere im Hinblick auf die Bedeutung 
von "Intelligenz". In der Diskussion über lernende und selbstständig handelnde Maschinen wird Intelligenz oftmals als zutiefst menschliche Eigenschaft beschrieben. Der Einbruch technischer Artefakte in diese Domäne hat noch immer etwas Unerhörtes, das das menschliche Selbstverständnis in Frage stellt. Wenn der Mensch die Krone der Schöpfung ist, muss er selbstverständlich auch die Krone der Intelligenz sein. Dabei zeigt sich, dass es der Mensch aufgrund seiner Entstehungsgeschichte gewohnt ist, sich zu anderen intelligenten Wesen zu verhalten. So sind bestimmte Lebewesen dem Menschen in spezifischen neuronalen Aufgaben, z. B. der Verarbeitung visueller Reize, ebenbürtig oder sogar überlegen. Und jede dieser Formen der Intelligenz hat ihre Daseinsberechtigung und verschwindet nicht angesichts einer höheren Entwicklungsstufe. Es existiert ein kontinuierlicher Wandel, der von horizontaler und vertikaler Vielfalt und Ko-Existenz geprägt ist. Der Mensch schafft sich somit auch durch die "Entfesselung der KI" nicht selber ab, sondern verändert sich, wie er es bereits während seines gesamten Daseins im Wechselverhältnis mit seiner Umwelt getan hat. Die Intelligenz wandelt sich ebenfalls, sie wechselt das Substrat von in vivo zu in silica, von Analytik zur Kreativität und so weiter.

So inspirierend dieser Ansatz auch ist, so stößt er hinsichtlich der Analyse von KI und Gesellschaft auch an Grenzen. Soziale Fragestellungen, wie diejenigen nach Machtverhältnissen unter den unterschiedlichen Akteuren innerhalb des Innovationsgeschehens Kl, fallen unter den Tisch. Für eine analytische Beschreibung der Beziehungen zwischen Mensch und KI reicht das Vokabular dieser von der Biologie und ihren Evolutionsprinzipien inspirierten Perspektive nicht. Zudem verleitet diese Sichtweise auch zu einem gewissen Fatalismus, der die Geschichte der KI sich selbst überlassen würde. Wann immer die Rolle der KI in der Gesellschaft, in der Arbeitswelt etc. thematisiert wird, ist ein Vergleich mit der Einzigartigkeit des Menschen nicht fern. Tatsächlich jedoch ist die meiste KI bei alltäglichen Aufgaben - ohne ihren Wert in irgendeiner Weise mindern zu wollen - viele Stufen darunter angesiedelt. Es handelt sich um kleine, beschränkte Aufgaben („tasks"), die keine Singularität, also ein technisches System mit Bewusstsein, benötigen und folglich auch gar nicht darauf abzielen, eine solche zu entwickeln. Es geht vielmehr um ein "Mitdenken" bei diesen Aufgaben - also darum, eine Aufgabenstellung zu erkennen, das Vorher und Nachher vorausschauend zu vergleichen, sowie Lösungsmöglichkeiten wie Werkzeuge und Material oder eigene Fähigkeiten, zu überprüfen. Der Anspruch, der universellen Intelligenz des Menschen zu gleichen, wird dabei mit hoher Wahrscheinlichkeit eher selten gefordert sein. Intelligente Maschinen müssen vielmehr Bauteile richtig auswählen und platzieren, Systemparameter in Abhängigkeit von internen und externen Einflussfaktoren einstellen und Situationen anhand von Indikatoren wie Verkehr oder Krankheiten erfassen können. Gegenwärtig besteht also weniger die Gefahr, dass uns die $\mathrm{KI}$ in den kommenden Jahren überfordert und überflügelt, sondern vielmehr darin, dass wir unerfüllbare Erwartungen an die technischen Systeme haben. 
Die Beiträge in diesem Teil tragen der Vielseitigkeit des Innovationsphänomens KI Rechnung, weil ihre analytischen Ansätze ähnlich vielfältig sind. Gemeinsam ist ihnen der Fokus auf die Wechselbeziehungen zwischen Gesellschaft, Wirtschaft, Politik und Technologie. Sie richten den Blick auf die sozialen Arenen, in denen das Innovationspotenzial von $\mathrm{KI}$ ausgehandelt wird, darauf, wer an diesen Prozessen beteiligt ist und welche sozialen und technischen Pfadabhängigkeiten existieren. Sie entwerfen Szenarien, wie in der Gesellschaft KI gestaltet werden kann und unter welchen Bedingungen KI demokratisch akzeptabel ist.

In der Beschreibung der Mikro-Konstellationen sehen die Autoren der Beiträge genau wie Serebriakoff Mensch und Technik nicht als isolierte Sphären. In der Gesamtschau treten drei Muster hervor, die auf gesellschaftlicher Ebene die Beziehungen zwischen Menschen und KI charakterisieren. Diese Muster sind nicht trennscharf, sondern bauen aufeinander auf.

\section{Erstes Muster: KI ist ein Bestandteil der Gesellschaft.}

Diese Aussage ist eine grundsätzliche Diagnose und impliziert eine analytische Haltung: Gesellschaft und KI können nicht als zwei entkoppelte Sphären gedacht werden. $\mathrm{Kl}$ ist keine Innovation, die in einem geschlossenen Labor entwickelt und nach Vollendung freigelassen wird, um dort auf die Gesellschaft zu treffen. Die Innovation "KI" folgt vielmehr der Logik eines „Realexperiments", in dem eine Technologie, ohne ausgereift zu sein und ohne ihre Implikationen vorab bestimmt zu haben, angewandt wird. Der Sinn und Zweck von KI ergibt sich erst durch ihren Gebrauch, wenn sie mit sozialen Praktiken, gesellschaftlichen Werten und Lebenswelten gekoppelt wird.

Wir sind in einem Stadium angelangt, in dem sich Gesellschaft und KI nicht mehr unabhängig voneinander entfalten. Neue soziale Muster entstehen durch die Verschränkung gesellschaftlicher und technologischer Entwicklungen. Die empirischen Beispiele der Beiträge dieses Teils zeigen, dass KI ein prägender Bestandteil der Gesellschaft ist. Sie gehört zu unserem Alltag und wirkt darauf ein, wie wir arbeiten und miteinander kommunizieren. Ein Beispiel ist der Einsatz von $\mathrm{KI}$ in sozialen Medien: Algorithmisch sortierte Informationen beeinflussen politische Wahlen, und gleichzeitig verschafft soziale Kommunikation im Internet der KI ein wesentliches Feld für Innovationen. Der Beitrag 13 legt dar, wie KI im Rahmen von kreativer Arbeit selbst zum Ko-Schöpfer neuer Gegenstände und Medien wird. Hier rückt KI vermeintlich stark in das Hoheitsgebiet des Menschen vor, relevant wird sie jedoch erst, indem sie die gesellschaftliche Wertschätzung von Neuheit und Ästhetik nährt. Es ist somit durchaus nicht trivial festzustellen, dass KI integraler Bestandteil von Gesellschaft ist. 


\section{Zweites Muster: KI ist eine Herausforderung für die Gesellschaft.}

Wenn KI Bestandteil von Gesellschaft ist, kann diese mit ihr auch verändert werden, und zwar im Guten wie im Schlechten. Das macht KI zu einer großen gesellschaftlichen Aufgabe, denn sie kann demokratische Werte unterstützen, aber sie auch untergraben.

Die folgenden Beiträge geben Einblicke, wie sich diese Herausforderungen in einzelnen Bereichen zeigen und wie verschiedene Akteure über Chancen und Risiken verhandeln. Wenn es um Arbeit geht, werden die Möglichkeiten und Gefahren besonders stark und kontrovers diskutiert. Einerseits macht KI Arbeitsprozesse einfacher, flexibler und innovativer, andererseits drohen Arbeitsplatzverluste, die insbesondere die Mitte der Gesellschaft treffen würde. Die im Beitrag 11 angesprochene Polarisierungshypothese spitzt dies zu: KI wird das mittlere Qualifikationssegment automatisieren, während dies für Jobs am unteren Ende des Qualifikationsniveaus zu teuer ist und Tätigkeiten am oberen Ende weiterhin nicht technisch ersetzt werden können. Bei kreativer Arbeit entsteht eine ähnliche Ambivalenz, denn KI wird zum kreativen Impulsgeber, einer vormals exklusiv menschlichen Rolle.

Diese und weitere Beispiele zeigen, dass Menschen im Zusammenleben mit KI ihre Rollen oftmals erst finden müssen. Menschen verschwinden nicht einfach, das lehrt uns spätestens Serebriakoffs Bild der Evolution, aber die Gesellschaft steht vor der Aufgabe, Mutation und Selektion aktiv zu gestalten und durch einen entsprechenden Ausgleich dafür zu sorgen, dass sich kein naturgesetzliches "Survival oft he fittest" herausbildet.

\section{Drittes Muster: KI ist ein Spiegel der Gesellschaft.}

Die Einsicht, dass KI sowohl Bestandteil als auch Herausforderung von Gesellschaft ist, wirft oftmals mehr Fragen auf als Antworten bereitstehen. Einige davon gehen über den Sinn und Zweck spezifischer KI-Anwendungen hinaus und betreffen das menschliche Selbstverständnis: Wie viel KI wollen wir? Und wieso ist es für den Menschen überhaupt ein Problem, wenn es intelligentere Entitäten als ihn selbst gibt? Mit diesen Fragen wird KI zum Spiegel der Gesellschaft.

Die Autoren der Kapitel zur Ethik der KI diskutieren diese und weitere Fragen. Sie zeigen, dass mit der Einführung von KI oftmals Entscheidungsprobleme einhergehen, in denen Richtig und Falsch nicht einfach zu erkennen sind, sondern ethisch reflektiert werden sollten. Dies betrifft z. B. den Umgang mit persönlichen Daten. Diese ermöglichen erst den Einsatz von $\mathrm{KI}$, aber das richtige $\mathrm{Maß}$ an Datenfreigabe ist nicht einfach festzustellen. Daraus ergibt sich die Frage, welche Persönlichkeitsmerkmale des Menschen durch den Prozess der Digitalisierung zu technischen Nennwerten werden. Ähnliches gilt für das Problem, wie viel Kontrolle an KI übertragen werden 
darf: Wer trägt die Verantwortung für Entscheidungen? Was ist überhaupt Verantwortung in einem komplexen soziotechnischen System? Dies betrifft das Individuum sowie die Gesellschaft als Ganzes. Im Umfeld von Arbeit ist es aufschlussreich zu wissen, wie sich individuelle Kontexte verändern, was überhaupt als "Wert" menschlicher Arbeit zählt und inwiefern KI die sogenannte Arbeitsgesellschaft zu einem überholten Modell macht. Wenn KI als Kampfansage an etablierte Berufsbilder wahrgenommen wird, kann man umgekehrt auch fragen, wieso Arbeitslosigkeit ein Problem und nicht einen Erfolg darstellt - und ob der Fehler eher darin besteht, Arbeit weiterhin als konstituierenden Wert unserer Gesellschaft zu sehen. Ein ähnlicher Spiegel wird der Gesellschaft auch im Zuge künstlicher Kreativität (KK) vorgehalten, wenngleich aus anderer Richtung: Wenn Maschinen kreativ werden, tritt dann der Mensch eines seiner letzten Hoheitsgebiete an die Technik ab? Wieso ist das überhaupt problematisch? Verbirgt sich dahinter nicht ein sehr anthropozentrisches Weltbild, mit dem Menschen als einzig schöpferischem Wesen?

Nicht jedes der in den Beiträgen dieses Teils vorgestellten Beispiele kann in dieser Weise zu Grundsatzdiskussionen führen. Es wird jedoch deutlich, dass KI in ihrem Verhältnis zur Gesellschaft eine besondere Technologie ist. Ihre technische Funktionalität, die von bildhaften Vorstellungen des Menschseins geprägt ist, sowie ihr Eindringen in verschiedenste Gesellschaftsbereiche konfrontieren die Allgemeinheit nur allzu oft mit sich selbst.

Dabei bleiben die Autorinnen und Autoren der Beiträge dieses Teils nicht bei der Analyse und Beschreibung der vielfältigen Beziehungen zwischen KI und Gesellschaft stehen. Vielmehr schauen sie voraus und leiten Möglichkeiten ab, wie Forschung, Entwicklung und Innovation sozial gestaltet werden können. Die Vorschläge sprechen sowohl die öffentliche Regelung von KI im Hinblick auf Forschung und Anwendung an als auch die Wirtschaft bis hin zur einzelnen Person.

Da die Digitalisierung auf dem Weg zu sein scheint, zu einer "Technoreligion" zu werden, und die $\mathrm{KI}$ in dieser der vorläufige heilige Gral ist, verspricht auch die KI gleichermaßen Verdammnis und Erlösung. Das Ganze erinnert ein wenig an jene Diskussion, die im Jahr 1996 mit dem Schaf "Dolly" ihren Anfang nahm. Auch mit Blick auf das reproduktive Klonen standen die Zeichen der Debatte alsbald auf Apokalypse. Heute, rund 20 Jahre nach "Dolly", haben sich die technischen Möglichkeiten und mit ihnen die Erfolgsraten des Klonens verbessert. Jedoch hat gleichzeitig ein breiter Diskurs darüber stattgefunden (und dauert an), welches Leben unter welchen Voraussetzungen geklont werden darf. Bisher hat der Mensch nicht dazugehört. Natürlich ist der Vergleich zwischen KI und Klonen nur bedingt tauglich, denn das Erstellen von Quellcode und Algorithmen für eine $\mathrm{KI}$ ist etwas anderes als das Manipulieren von Zellen mit dem Ziel, dass sich diese zu einem vollständigen Menschen ausdifferenzieren. Aber dennoch verbindet beides die Frage nach der Einzigartigkeit 
des Menschen: Beim Klonen in individueller Hinsicht, bei der KI mit Blick auf das eigene Selbstverständnis. Und da ein sich dynamisch optimierender Programmcode in vielerlei Hinsicht dem genetischen Code und seiner evolutiven Veränderung ähnelt, kommt abermals die von Serebriakoff ins Spiel gebrachte Weiterentwicklung von biologischer und künstlicher Intelligenz zum Tragen. Da der Mensch gleichermaßen Subjekt und Objekt in der Entwicklung der KI sein kann, stellt sich im Kern die Frage nach der gesellschaftlichen Verantwortung. Unabhängig vom Typ der handelnden Person - ob im öffentlichen oder wirtschaftlichen Raum, ob im Gesamtgefüge oder individuell - muss jeder im Rahmen des eigenen Gestaltungspielraums Verantwortung tragen. Und vielleicht wird künftig eine wie auch immer geartete KI einer der zu beteiligenden Akteure in diesem Aushandlungsprozess sein.

\section{(c) (1)}

Dieses Kapitel wird unter der Creative Commons Namensnennung 4.0 International Lizenz http://creativecommons.org/licenses/by/4.0/deed. de) veröffentlicht, welche die Nutzung, Vervielfältigung, Bearbeitung, Verbreitung und Wiedergabe in jeglichem Medium und Format erlaubt, sofern Sie den/die ursprünglichen Autor(en) und die Quelle ordnungsgemäß nennen, einen Link zur Creative Commons Lizenz beifügen und angeben, ob Änderungen vorgenommen wurden.

Die in diesem Kapitel enthaltenen Bilder und sonstiges Drittmaterial unterliegen ebenfalls der genannten Creative Commons Lizenz, sofern sich aus der Abbildungslegende nichts anderes ergibt. Sofern das betreffende Material nicht unter der genannten Creative Commons Lizenz steht und die betreffende Handlung nicht nach gesetzlichen Vorschriften erlaubt ist, ist für die oben aufgeführten Weiterverwendungen des Materials die Einwilligung des jeweiligen Rechteinhabers einzuholen. 